\title{
Aggregation Induced Emission of
}

\section{Tetraphenylethylene into Styrene-based}

\section{Polymers}

Giuseppe Iasilli, ${ }^{a}$ Antonella Battisti, ${ }^{b}$ Francesco Tantussi, ${ }^{c}$ Francesco Fuso, ${ }^{c}$ Maria Allegrini, ${ }^{c}$ Giacomo Ruggeri, ${ }^{a}$ Andrea Pucci ${ }^{a,}$

${ }^{a}$ Dipartimento di Chimica e Chimica Industriale, Università di Pisa, Pisa, Italy

${ }^{b}$ NEST, Scuola Normale Superiore and Istituto Nanoscienze-CNR, Pisa, Italy

${ }^{\mathrm{c}}$ Dipartimento di Fisica, Università di Pisa, Pisa, Italy

Corresponding author:

Dr. Andrea Pucci, Dipartimento di Chimica e Chimica Industriale, Università di Pisa, Via Risorgimento 35, 56126 Pisa, Italy

Tel: +390502219270

Fax: +390502219260

e-mail: apucci@dcci.unipi.it 


\begin{abstract}
The present work outlines the preparation of different styrene-based polymer films containing small amounts (0.005-0.1 wt.\%) of tetraphenylethylene (TPE) fluorescent molecular rotors and the evaluation of their photoluminescent behaviour. Poly(styrene) (PS), poly(styrene- $b$ butadiene- $b$-styrene) (SBS) and poly(styrene-co-butadiene) (SBR) were selected as polymer matrices and their blend films were obtained after compression moulding with an uniform thickness $(150-200 \mu \mathrm{m})$.

The investigations demonstrated that when TPE was dispersed in a poor solvent or in the glassy PS matrix, the intramolecular rotations of its aryl rotors resulted completely arrested, thus favouring the strong emission of light centred at about $470 \mathrm{~nm}$. On passing to SBS and eventually to SBR or in a good solvent for TPE, the fluorescence significantly weakened to pale green and then to an hardly detectable signal, being phenyl rings rotations around $\mathrm{C}-\mathrm{C}$ double bond more permitted. Near field optical microscopy of TPE/polymer films correlated the distinct average fluorescence intensities with the different matrix morphology.
\end{abstract}




\section{Introduction}

In the last decade, a new class of polymeric materials called functional materials ${ }^{1,2}$ has been rapidly evolved due to the development of modern technologies that require unusual combination of properties that can not be provided by polymers. Thermoplastic polymers are made of long flexible molecules, which are characterized by a backbone of atoms connected through single covalent bonds. This feature confers stability and flexibility properties, but is not suitable for obtaining colour and response to external solicitations, which are extremely attractive properties of modern materials. ${ }^{3-6}$ Recently, the dispersion of luminescent aggregachromic dyes into polymer films have been effectively used for the preparation of materials showing both typical thermoplastic polymer properties (viscoelasticity) and optical response to visible light. The realization of "built-in" temperature and deformation plastic optical indicators has been demonstrated by several authors. ${ }^{7,8}$ The basic principle of these chromogenic materials is founded on colour changes in absorption or in emission associated with the structural modifications of the molecular assemblies of dyes contained in the continuous polymeric phase. Following this mechanism, the conversion between the monomer and aggregated dye states upon external solicitations leads to distinguishable optical changes of the composite material. ${ }^{7,8}$

However, it is also known that aggregation of organic luminophores often leads to partial or even complete quenching of their light emissions. ${ }^{9}$ This aggregation-caused quenching (ACQ) effect has limited the scope of technological applications of the luminogenic molecules. Alongside the development of even more effective aggregachromic chromophores, a new phenomenon called Aggregation-Induced Emission (AIE) is rapidly expanding in literature as an effective tool able to relieve the ACQ effect in the condensed phase. ${ }^{10-17}$ The AIE phenomenon is based on the fact that luminogen aggregation played a constructive, instead of a destructive, role in the light-emitting process: a series of molecules 
were found to be non-luminescent in the solution state but emissive in the aggregated state. For example, tetraphenylethylene (TPE) is non-emissive in dilute solutions but becomes highly luminescent when their molecules result aggregated in concentrated solutions or cast into solid films. ${ }^{18,19}$ In detail, in a dilute solution, TPE undergoes dynamic intramolecular rotations against its double bond and renders its molecule non-luminescent. ${ }^{20,21}$ Conversely, in the aggregate state, the intramolecular rotations of its aryl rotors are greatly restricted, thus opening up the radiative channel. ${ }^{22}$ AIE was first realized in propeller-like small molecules and only very recently extended to polymeric systems which exhibit an array of functional properties which suggest potential applications as explosive chemosensors, light-emitting materials, including bioprobes for in vitro and in vivo imaging applications. ${ }^{14,23-27}$ Inspired by this idea, in this paper, we applied the photophysics of TPE to thermoplastic styrene-based polymers and explored its ability to discriminate the host polymer matrix in terms of its distinct composition, architecture and inherent viscosity. For this purpose, TPE was mixed with three different thermoplastic matrices, i.e. poly(styrene) (PS), poly(styrene$b$-butadiene- $b$-styrene) (SBS) and poly(styrene-co-butadiene) (SBR) at different concentration (from 0.005 to 0.1 wt.\%), and its photophysics accurately investigated through spectroscopic and microscopic investigations. The results were discussed in terms of developing a new tool for polymer traceability. 


\section{Experimental part}

\subsection{Materials}

Tetraphenylethylene was supplied by Sigma-Aldrich and purified by recrystallization from a mixture of dichloromethane-methanol (2:1 v/v). Poly(Styrene- $b$-Butadiene- $b$-Styrene) (SBS, Polimeri Europa, 31\% w/w Sty, $\left.\mathrm{M}_{\mathrm{W}}=9300-41400-9300 \mathrm{~g} / \mathrm{mol}, \quad \delta=0.94 \mathrm{~g} / \mathrm{cm}^{3}\right)$, Poly(Styrene-co-Butadiene) (SBR, Polimeri Europa, 32\% w/w Sty, Mooney Viscosity ML $\left.1+4\left(100{ }^{\circ} \mathrm{C}\right)=49\right)$, atactic Polystyrene (PS, Repsol, $\left.\mathrm{M}_{\mathrm{W}}=86000 \mathrm{~g} / \mathrm{mol}\right)$ and Poly $(1,4-$ Butadiene) (PB, Sigma-Aldrich, $\mathrm{M}_{\mathrm{W}}=5000 \mathrm{~g} / \mathrm{mol}$ ) were used as received. All used solvents were reagent or spectroscopic grade (Carlo Erba or Aldrich) and utilised without further purification.

Preparation of TPE solutions

Since TPE is sensitive to UV radiation and oxidizers, ${ }^{28} 10^{-4}-10^{-6} \mathrm{M}$ TPE solutions in hexane, dioxane, toluene and chloroform were prepared after deaerating the solvent by bubbling with nitrogen for 30 minutes, and stored away from light.

Preparation of polymeric mixtures

TPE/SBS and TPE/SBR blend films were prepared by dissolving $1 \mathrm{~g}$ of polymer and the desired amount of TPE in $150 \mathrm{~mL}$ of $\mathrm{CHCl}_{3}$. After solvent evaporation, the polymer mixture was melt-pressed between two Teflon foils in a Carver $3851-0$ press at $150{ }^{\circ} \mathrm{C}$ and 4 tons of pressure for 5 minutes. After removal from the press, the films (180-220 $\mu \mathrm{m}$ thick) were allowed to reach slowly room temperature. TPE/PB mixture was prepared by solution mixing in chloroform and solvent evaporation at low pressure.

\subsection{Apparatus and Methods}

UV-vis absorption studies of TPE solutions and polymer blend films were carried out at room temperature with the help of a Perkin-Elmer Lambda 650. Steady-state fluorescence spectra 
were acquired under isotropic excitation at $360 \mathrm{~nm}$, with the help of a PerkinElmer luminescence spectrometer LS55 controlled by FL Winlab software and equipped with the front surface accessory. The fluorescence quantum yield $(\Phi)$ of TPE solutions and polymer films was determined relative to quinine sulfate $\left(\Phi^{\mathrm{S}}=0.54\right.$ in $\left.0.1 \mathrm{M} \mathrm{H}_{2} \mathrm{SO}_{4}\right)$ using the following relation ${ }^{29}$

$$
\Phi_{f}=\Phi_{f}^{s} \frac{\int_{0}^{\infty} I_{F}(v) d v}{\int_{0}^{\infty} I_{F}^{S}(v) d v}\left(\frac{1-10^{-A^{s}}}{1-10^{-A}}\right) \frac{n^{2}}{n_{s}^{2}}
$$

where $\Phi_{f}^{s}$ is the quantum yield of standard and the integrals are the area under fluorescence peaks. $A$ and $A^{\mathrm{S}}$ are the absorbances of the molecule and standard, respectively, at the excitation wavelength $(360 \mathrm{~nm}) . n$ is the refractive index of the medium.

The refractive index of Water, Dioxane, Polystyrene, SBS and SBR are respectively $1.3330,{ }^{30}$ $1.4224,{ }^{30} 1.5894,{ }^{31} 1.5300,{ }^{31} 1.5350 .{ }^{31}$ Refractive index of water-dioxane mixtures were calculated on the weighted average of the two components according to their molar fraction. In the analysis of fluorescence variation with temperature, the time evolution of the emission at $465 \mathrm{~nm}$ of a TPE/PB sample was approximately correlated with temperature by applying the Newton's law of heating. ${ }^{32}$

Scanning Near-field Optical Microscopy (SNOM) analysis has been performed using a custom-made instrument able to map the optical properties of the samples with subwavelength spatial resolution. ${ }^{33-35}$

The instrument operates in the emission-mode: the sample surface interacts with the nearfield produced by a tapered optical fiber probe (Nanonics Imaging, Ltd.) featuring an apical aperture $50 \mathrm{~nm}$ wide (nominal). Excitation is accomplished by using a semiconductor laser at $\lambda=405 \mathrm{~nm}$ producing, in typical operating conditions, a near-field power on the order of tens of $\mathrm{nW}$. The fluorescence emission from the sample surface is collected in the far-field by a long distance microscope objective (Mitutoyo Apo-Plan 10X) and directed onto a 
miniaturized photomultiplier (Hamamatsu R-5600). In order to cut contribution from elastic scattering and reflection of the laser light, a pair of spectral filters (Melles-Griot LPF450) is placed in front of the detector, ensuring a rejection factor above six orders of magnitude for the $405 \mathrm{~nm}$ laser radiation while being highly transparent in the wavelength range of the fluorescence (above $450 \mathrm{~nm}$ ). The fluorescence map, comprising of typical 256x256 pixels, is then reconstructed point by point during a raster scan of the sample. Simultaneous acquisition of a topography map is accomplished by using the shear-force method, ${ }^{35}$ which is also responsible for keeping the probe-to-surface distance within the near-field range (typically, the distance is $5-10 \mathrm{~nm}$ ). In addition, a miniaturized photomultiplier placed below the sample collects the optical signal transmitted by the sample, leading to produce optical maps representative of the material absorption and of the coupling efficiency between the optical near-field and the sample surface. Such maps are mostly used for reference and check purposes, aimed in particular at assessing a constant efficiency of the near-field coupling to the material. Signal-to-noise ratio in the optical maps has been improved by using lock-in amplifiers referenced to amplitude modulation of the excitation laser intensity.

Figure S1 (supplementary info) shows a sketch of the experimental configuration.

Lifetime parameters were collected by using a Leica TCS SP5 SMD inverted confocal microscope (Leica Microsystems AG, Wetzlar, Germany) equipped with an external pulsed diode laser (PicoQuant GmbH, Berlin, Germany) for excitation at $405 \mathrm{~nm}$. Laser repetition rate was set to $40 \mathrm{~Hz}$. Image size was $256 \times 256$ pixels and scan speed was usually set to 400 $\mathrm{Hz}$ (lines per second). The pinhole aperture was set to 0.5 Airy. Polymeric films fixed on glass slides were viewed with a 100×, 1.3 NA oil immer-sion objective (Leica Microsystems). The images were collected using low excitation power at the sample (10-20 $\mu \mathrm{W})$. Emission was monitored in the $430-540 \mathrm{~nm}$ range by acousto-optical tunable beam splitters (AOBS)-based built-in detectors. Acquisition lasted until about 100-200 photons per 
pixel were collected, at photon counting rate of 100-500 kHz. Lifetime images were elaborated using Picoquant SymPhoTime and FluoFit softwares for FLIM analysis.

\section{Results and discussion}

\subsection{Optical characterization of tetraphenylethylene solutions}

Tetraphenylethylene (TPE) showed UV absorption in the near UV-range (320-400 nm) due to the $\mathrm{S}_{0}-\mathrm{S}_{1}$ transition with an absorption peak centred at $310 \mathrm{~nm}$ (i.e., $309 \mathrm{~nm}(\varepsilon=12100$ $\left.\mathrm{M}^{-1} \mathrm{~cm}^{-1}\right)$ for dioxane, $310 \mathrm{~nm}\left(\varepsilon=13800 \mathrm{M}^{-1} \mathrm{~cm}^{-1}\right)$ for hexane and $311 \mathrm{~nm}(\varepsilon=12600$ $\mathrm{M}^{-1} \mathrm{~cm}^{-1}$ ) for toluene). TPE solutions were practically non-luminescent or showed very weak emission or light diffusion phenomena. It has been already reported that the optical properties of TPE depend on its molecular shape and, more specifically, on the slightly twisted conformation of its ground state. ${ }^{20}$ Semiclassical dynamics simulation has revealed that the length of the ethylenic bond is typical of a C-C double bond, but the four vinyl-phenyl bonds are not coplanar, and dihedral angles of approximately $50^{\circ}$ are actually formed between the planes containing each phenyl ring and the one containing the central double bond. This peculiarity confers to the molecule the typical shape of a four-bladed propeller. In good solvents, TPE phenyl rings are freely to rotate, whose motions effectively quench the luminescence of the molecule.

Conversely, when large amounts of water ( $>60$ vol.\%) were added to $10^{-5} \mathrm{M}$ dioxane solutions (with final concentrations kept unchanged), the resultant mixtures showed intense fluorescence centred at $460 \mathrm{~nm}$, which progressively shifted to higher wavelenghts (Figure 1a). The addition of water, that is a poor solvent for TPE, forced TPE molecules to aggregate, thus blocking the motion of phenyl rings, and eventually favouring the radiative process. For water contents of about $65-75$ vol.\%, TPE molecules assembled in regularly stacked emissive assemblies with a quantum yield (QY) of about 0.14 (Figure 1b). For water contents $>80$ 
vol.\%, the significant reduction of the QY was attributed to a combination of effects: very large amounts of water in the mixture induced TPE aggregation in a poorly ordered structures characterized by lower emission; also, these supramolecular dye structures caused substantial light diffusion phenomena and the displacement of the entire absorption spectrum to higher absorbance values, which favoured self-absorption phenomena (Figure 1c). This was also the reason why the emission peak shifted progressively to higher wavelengths (Figure 1a).

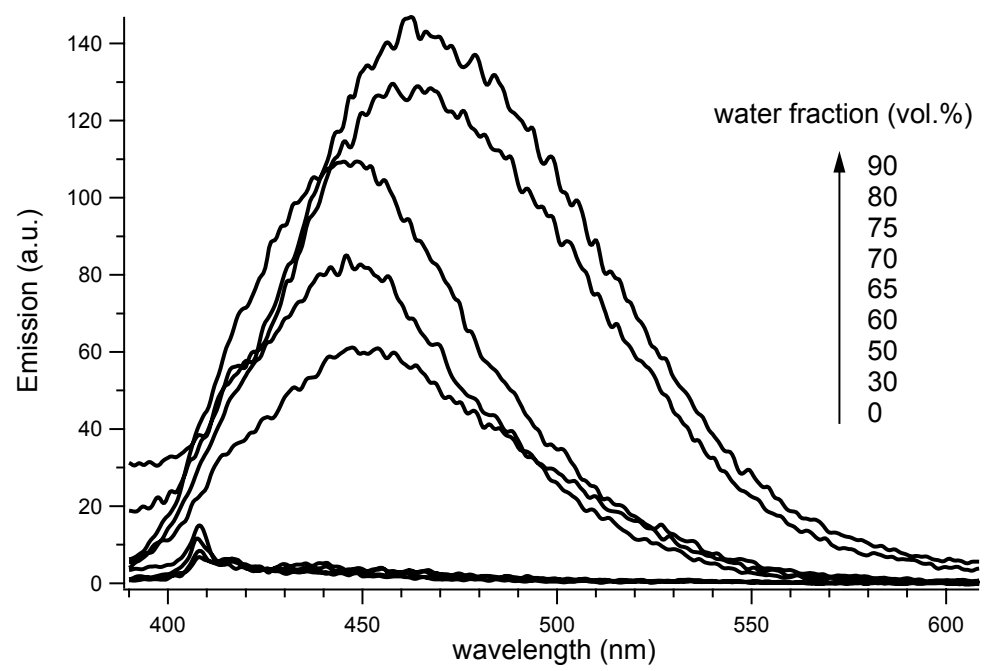

(a)

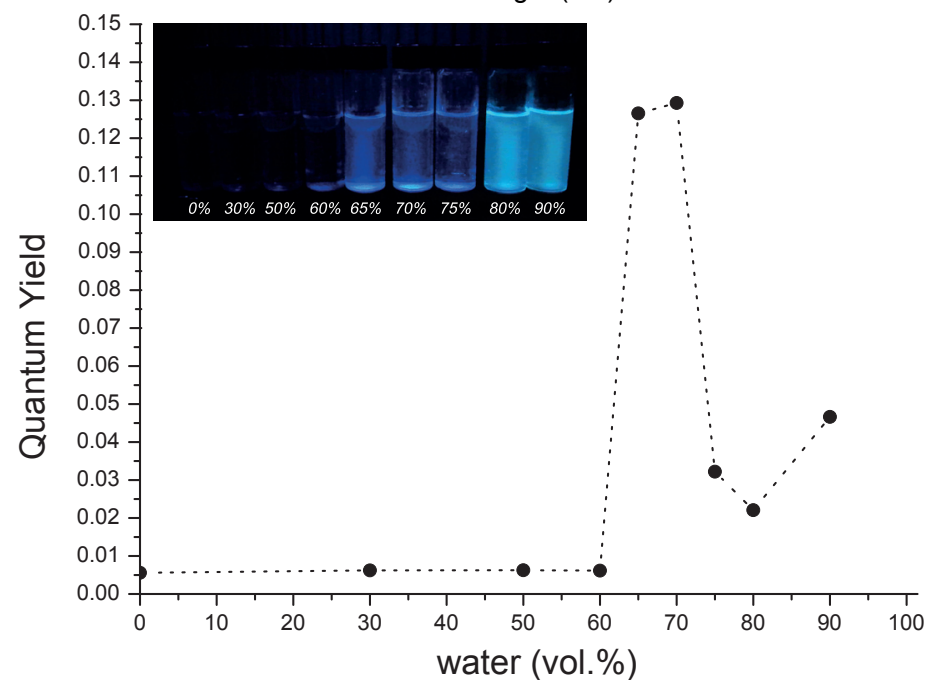

(b) 


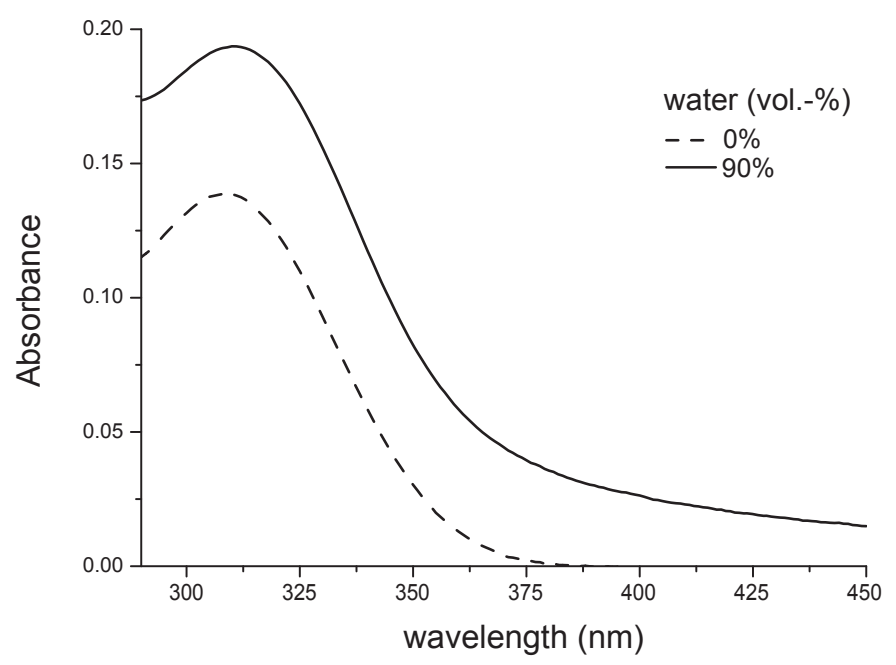

(c)

Figure 1. a) Fluorescence spectra (exc. $=360 \mathrm{~nm})$ of TPE dilute solutions $\left(10^{-5} \mathrm{M}\right)$ in dioxane/water mixtures with different water contents; $b$ ) dependence of quantum yield on the

water content in dioxane/water mixtures and (inset) a photograph of the sample under irradiation with UV light at $366 \mathrm{~nm}$; c) UV-vis absorption spectra of TPE dilute solutions (10$\left.{ }^{5} \mathrm{M}\right)$ in pure dioxane $(0 \%)$ and in in dioxane/water mixture containing the 90 vol.\% of water.

\subsection{Optical characterization of tetraphenylethylene/polymer films}

TPE/polymer films (0.005-0.1 wt.\%) having thickness of 150-200 $\mu \mathrm{m}$ were prepared by compression moulding of mixtures obtained after evaporating dye/polymer chloroform solutions. Copolymers of styrene and butadiene with similar composition (Styrene $=31 \mathrm{wt} . \%$ for SBS and 33 wt.\% for SBR) but different thermal properties were utilised. More specifically, SBS was a thermoplastic elastomer whose triblock-structure combines the properties of the hard segment polystyrene blocks $\left(\mathrm{T}_{\mathrm{g}}=70{ }^{\circ} \mathrm{C}\right)$ and the soft-segment polybutadiene blocks $\left(\mathrm{T}_{\mathrm{g}}=-87^{\circ} \mathrm{C}\right),{ }^{36,} 37$ whereas $\mathrm{SBR}$ was a random styrene/butadiene copolymer with a $\mathrm{T}_{\mathrm{g}}$ of $-21^{\circ} \mathrm{C} .^{38}$

All dye/polymer films showed superimposable UV-Vis absorption spectra, with absorption maximum centred at about $310 \mathrm{~nm}$, as analogously observed for TPE solutions. Conversely, all the films displayed fluorescence in the range between 400 and $550 \mathrm{~nm}$, with an increasing trend along the series from SBR to PS (Figure 2). 


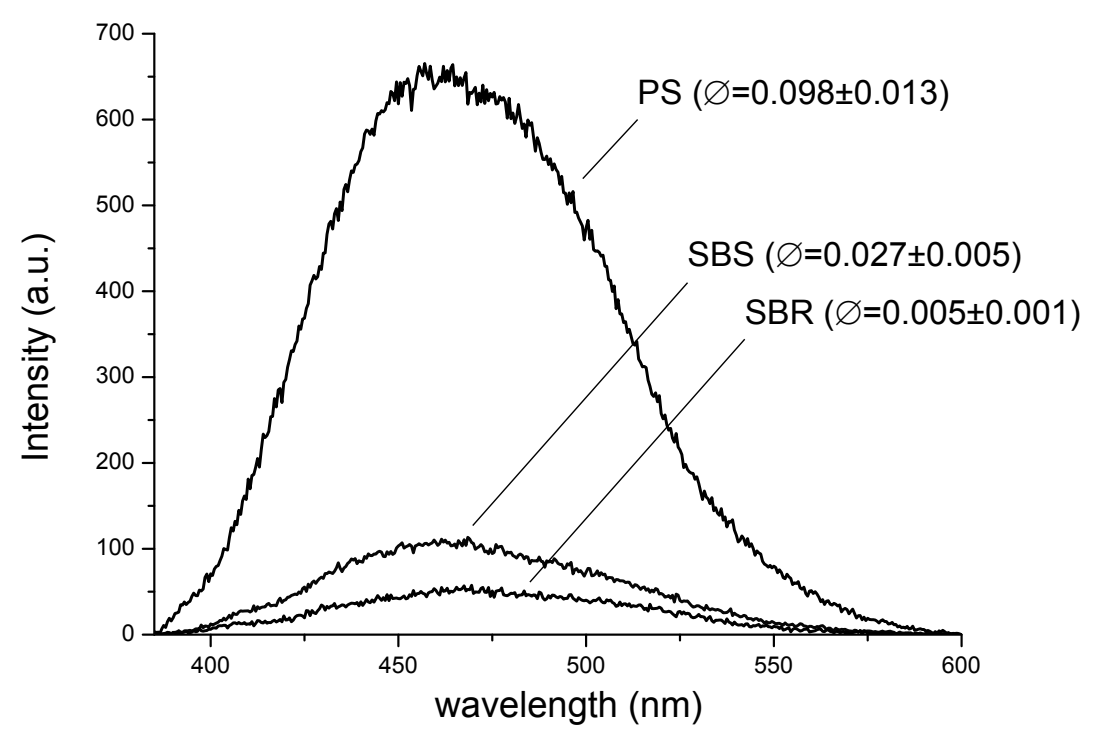

Figure 2. Emission spectra of $0.01 \mathrm{wt} . \%$ TPE/polymer films and their fluorescence quantum yields

The reason of the distinct emission intensities between the different films was ascribed to the nature of the continuous polymer matrix. For example, when TPE was dispersed in the glassy amorphous matrix of PS $\left(\mathrm{T}_{\mathrm{g}}=70{ }^{\circ} \mathrm{C}\right)$, the intramolecular rotations of its aryl rotors resulted completely arrested, thus favouring the strong emission of light centred at about $470 \mathrm{~nm}$. On passing to SBS as the polymer matrix, the fluorescence significantly weakened. This phenomenon was addressed to the amount of TPE dispersed in the highly viscous, but not glassy, butadiene block $\left(\mathrm{Tg}=-87^{\circ} \mathrm{C}\right)$ in which the dye results less emissive, being phenyl rings rotations around $\mathrm{C}-\mathrm{C}$ double bond more permitted. This finding was confirmed by analysing the faint fluorescence coming from TPE/SBR films. The SBR matrix is characterised by a $\mathrm{T}_{\mathrm{g}}$ of $-21{ }^{\circ} \mathrm{C}$ and the contained TPE molecules therefore behaves like in solution, showing poor, even if detectable, luminescence $(\varnothing=0.005 \pm 0.001)$.

Moreover, the different luminescence between TPE/SBS and TPE/SBR films was also worth of noting (Figure 3). 


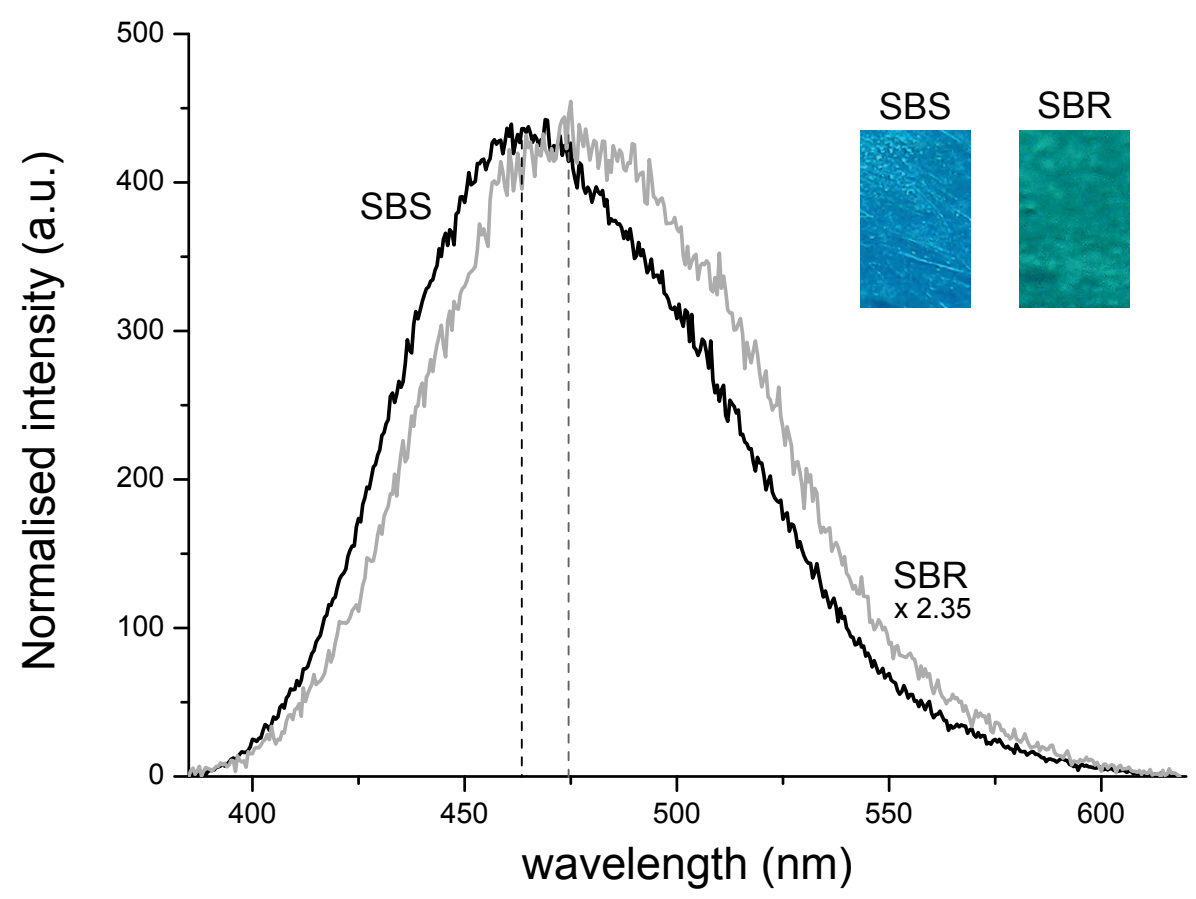

Figure 3. Normalised emission spectra of TPE/SBS (black line) and TPE/SBR (gray line) films containing the $0.05 \%$ of dye, and (inset) images of the films taken under the illumination of a long-range UV lamp at $366 \mathrm{~nm}$

TPE/SBS composites showed emission peaked at $460 \mathrm{~nm}$, whereas SBR films containing the same amount of TPE (0.05 wt.\%) displayed a less intense luminescence ( 2.5 times lower), and about $15 \mathrm{~nm}$ red-shifted from the former. This feature conferred to TPE/SBS films a brilliant blue emission, in contrast with the pale green luminescence characteristic of the SBR-based blend (Figure 3, inset).

According to the diagram of the complex TPE photophysics (Figure S1), the higher the viscosity of the medium, the greater the energy barriers to overcome to reach the more stable ${ }^{1} \mathrm{p}^{*}$ state, from which TPE decays by non-radiative pathways. ${ }^{21}$ Therefore, it can be assumed that when TPE is dissolved in the styrene phase, the only motions allowed to TPE are those related to the thermal relaxation, which enables the transition from $S_{1 V}^{*}$ to $S_{1 V}$, and the eventual emission of light at about $460 \mathrm{~nm}$. Conversely, in the butadiene phase or in the SBR matrix, TPE motions permit the molecule to overcome the potential barriers arising from the viscous, but not glassy medium, and to reach the excited states $S_{1 \mathrm{R}}$ and $1 \mathrm{p}^{*}$ from which it 
decays by emitting light at $530 \mathrm{~nm}$ or in a non-radiative way, respectively. Those explanations can possibly elucidate why TPE molecules dispersed in SBR emit light $\sim 15 \mathrm{~nm}$ red-shifted than that from PS or SBS films. In connection with these findings, TPE mixed with a polybutadiene matrix $\left(\mathrm{Tg} \sim-110{ }^{\circ} \mathrm{C}\right)$ showed negligible fluorescence at room temperature, whereas a brilliant and intense blue emission came up upon dipping the blend in liquid nitrogen (Figure 4).

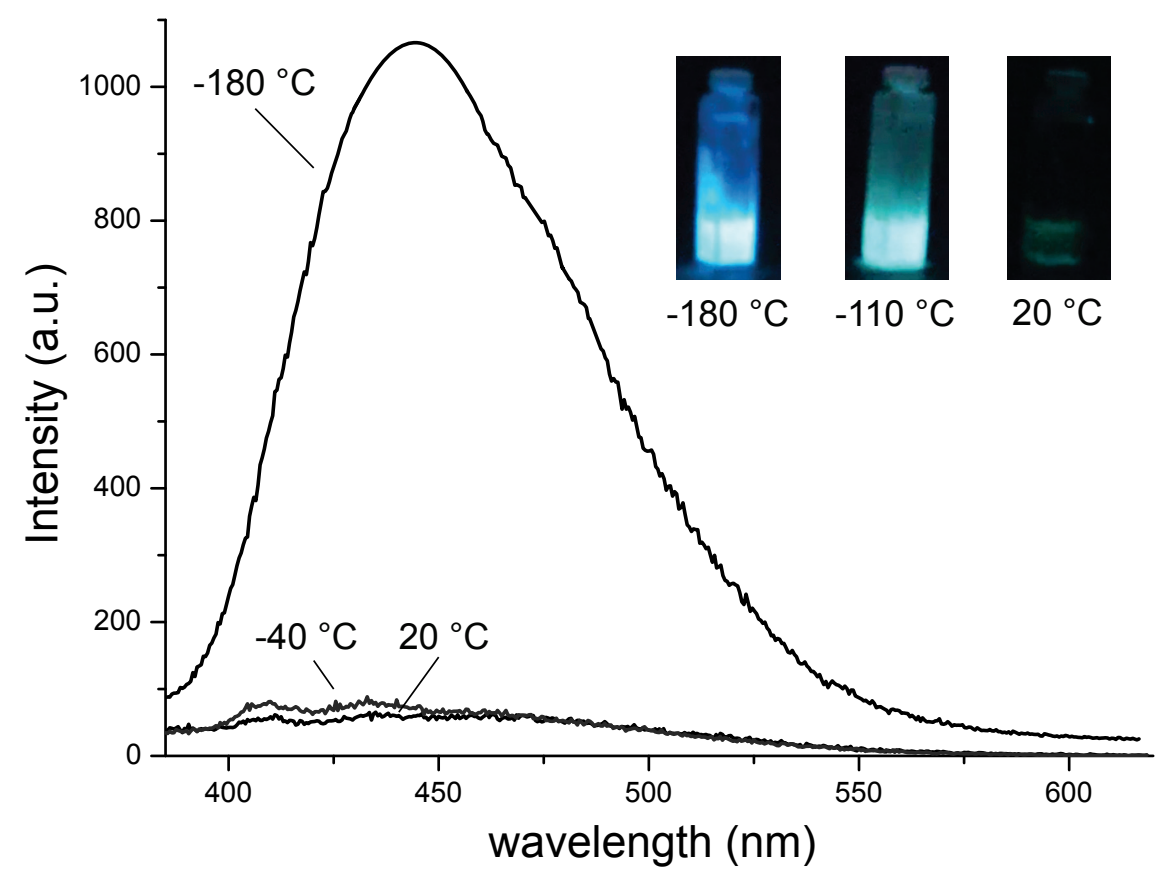

Figure 4. Emission spectra of a 0.01 wt.\% TPE/PB mixture at different temperatures after the immersion in a liquid nitrogen bath and images (inset) of the same mixtures taken under the illumination of a long-range UV lamp at $366 \mathrm{~nm}$. The time evolution has been approximately correlated with temperature by applying the Newton's law of heating ${ }^{32}$

The luminescence of TPE/SBR and TPE/SBS films containing the $0.05 \%$ of dye were also analysed by lifetime experiments. TPE/SBR mixtures were characterized by average lifetime parameters of 1.04 (62\% population) and $4.47 \mathrm{~ns}$ (38\% population), whereas TPE/SBS films showed longer-lived emission, i.e. 0.90 (45\% population), 3.31 (31\% population) and $7.17 \mathrm{~ns}$ (24\% population). This behaviour was in agreement with the fluorescence decay dynamics reported in literature for TPE solutions. ${ }^{21}$ In fact, more water content or the use viscous 
solvents result in much longer lifetime. In other words, more aggregated are TPE molecules and longer lifetime emission occurs.

\subsection{Scanning Near-field Optical Microscopy (SNOM) Characterization}

SBS and SBR samples containing TPE (0.05 wt.\%) were also deeply analyzed by near-field optical microscopy, with the main aim to observe the emission morphology that is the spatial distribution of the fluorescence intensity at the sample surface. For such analysis, excitation was accomplished with the near-field produced out of a tapered optical fiber probe coupled to $405 \mathrm{~nm}$ laser radiation. Such a wavelength falls in the tail of the absorption spectrum of TPE. Nonetheless, tests performed out of the SNOM, i.e., in macroscopic conditions, demonstrated it was able to excite weak fluorescence from the sample, with spectral properties similar to those achieved upon $366 \mathrm{~nm}$ light excitation (see, e.g., the insets in Figures 3 and 4).

Near-field fluorescence maps, spectrally integrated above $450 \mathrm{~nm}$, can indeed be recorded upon near-field excitation at that wavelength. In agreement with the macroscopic analysis, the average fluorescence intensity of the maps is smaller in TPE/SBR than in TPE/SBS by a factor 3-5, and this occurs in all investigated samples.

Due to the fabrication procedure, the surface of the thick films is affected by an inherent roughness on the order of $50-80 \mathrm{~nm}(\mathrm{rms})$ over a $100 \times 100 \mu \mathrm{m}^{2}$ area, related to the presence of small droplets, fractures, and holes. The corresponding topographical variations affect the fluorescence intensity acquired at the local scale, hampering reliable reconstruction of the emission morphology. In order to overcome such a limitation, we applied a derivative-filter procedure to the raw maps aimed at enhancing the small-scale variation of the intensity distribution with respect to the macro-scale variations associated with topographical changes. Moreover, we selected regions of the scan with a rather constant near-field transmission signal, ensuring that the coupling efficiency between the near-field and the sample surface is kept almost constant, as well. 
An example of the results is shown in Figure 5, reporting topography and fluorescence maps for three different kinds of sample. It is clear that in all of them fluorescence is not homogeneously distributed over the surface. This can be ascribed to the uneven distribution of the dye concentration within the matrix, in agreement with previously reported near-field observations of optical properties in luminescent $t^{39-41}$ and photoactive $\mathrm{e}^{34,42,43}$ host-guest systems.

The topography does not markedly depend on the matrix structure and shows always the already mentioned inherent roughness. We included in the analysis a TPE/SBS sample mechanically stressed through the application of an uniaxial drawing (drawing ratio around 3), whose representative maps are shown in panel (c). In this case, the mechanical drawing leads to a decrease in average roughness and to the appearance of micrometer-sized islands roughly aligned along the drawing axis (approximately placed along the diagonal of the maps shown in the figure).

Conversely, the fluorescence distribution turns to depend on the matrix structure. Besides the already mentioned larger average fluorescence intensity in the TPE/SBS samples (including the drawn one), morphology appears to be systematically affected by the either random or block structure of the matrix. Small, typically round-like, features, with a transverse size $(100-150 \mathrm{~nm})$ slightly larger than the expected instrumental resolution $(50 \mathrm{~nm}$, corresponding to the probe aperture size), are observed in all samples. We remark that observation of most of features is confirmed when performing repeated scans of the same portion of the sample, ruling out they are due to noise.

Such small fluorescence domains are randomly distributed and less contrasted in TPE/SBR, while they appear to coalesce each other in TPE/SBS, giving rise to broader, island-like features. Distribution of such islands is related to topography; in particular, mechanical drawing typically leads to several homogeneous islands aligned along the drawing axis. 
The uneven distribution of fluorescence domains in TPE/SBR is in agreement with the expectedly random distribution of dye molecules within the matrix. Moreover, the weak contrast associated to such features suggests that no specific mechanism is active in ruling the emission at the local scale. On the contrary, the presence of a block copolymer matrix is expected to drive the dye distribution in either the styrene or the butadiene blocks, the latter being disadvantaged in terms of emission intensity. The spatial resolution of our instrument (around $50 \mathrm{~nm}$ ) is not large enough to enable discrimination at the level of the single blocks. However, it is sufficient to reveal aggregates of blocks, i.e., regions at the surface where either styrene or butadiene blocks are mostly frequent, characterized by either stronger or weaker fluorescence yield. Remarkably, their occurrence is related, to some extent, with the topography that can be in turn associated to variations in the local mechanical stress produced during the compression molding process. Indeed, addiction of an artificial stress such as in uniaxial drawing promotes coalescence, i.e., broadening of the butadiene or styrene block containing regions.

(a)
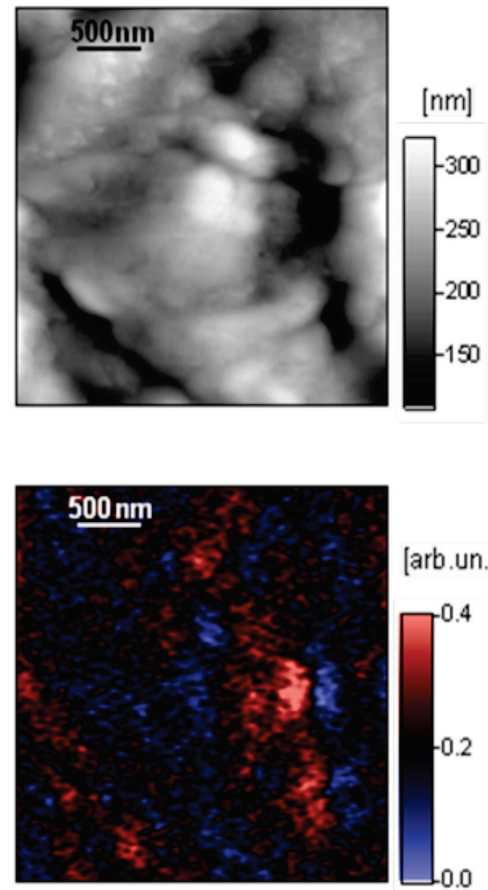

(b)
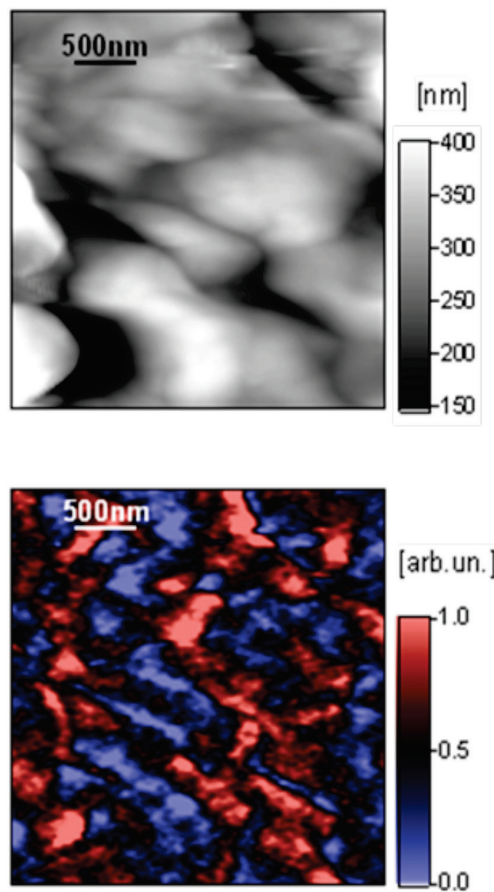

(c)
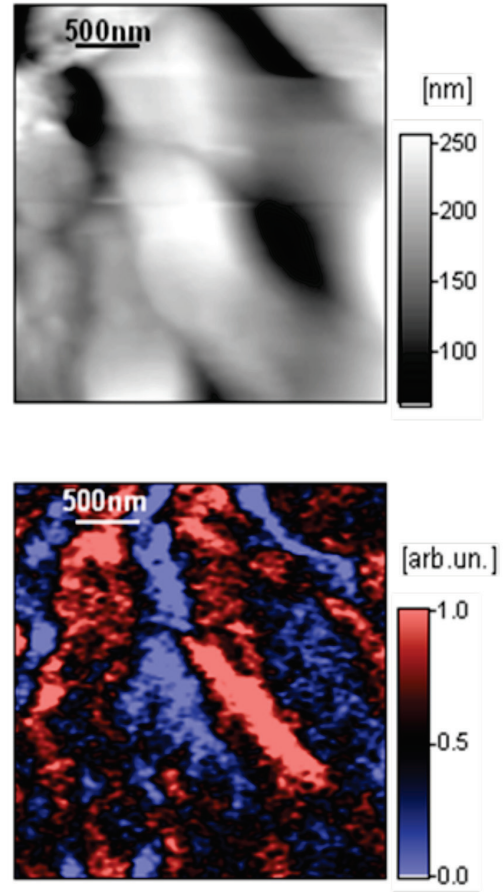
Figure 5. Topography maps on the top row and fluorescence maps at $\lambda>450 \mathrm{~nm}$, treated as described in the text, on the bottom row. Samples: (a) TPE/SBR; (b) TPE/SBS; (c) TPE/SBS upon uniaxial drawing (drawing ratio roughly 3 ) along a direction approximately lying on the diagonal of the map. Note the different scales, in arbitrary units, for the fluorescence map (a) compared to (b) and (c), that reflects the lower fluorescence intensity found in TPE/SBR with respect to TPE/SBS.

\section{Conclusions}

We have demonstrated that the fluorescence of TPE molecular rotors is basically dominated by the structural properties of the polymer matrix in which they are contained. More specifically, when TPE is dissolved in styrene-based polymers with different thermal and morphological properties, their emission result abruptly enhanced on passing from the rubbery highly viscous matrix of SBR to the totally glassy of PS. This effect stems from the photophysics of TPE which is strictly related to its propeller shape, since the rotation of the phenyl rings strongly affects its luminescence. When TPE was dispersed in PS, the intramolecular rotations of its aryl rotors resulted completely arrested, thus favouring the strong emission of light centred at about $470 \mathrm{~nm}$. On passing to SBS and eventually to SBR, the fluorescence significantly weakened to pale green and then to an hardly detectable signal, being phenyl rings rotations around C-C double bond more permitted.

In agreement with these results, SNOM maps of TPE/SBS samples showed larger average fluorescence intensities than the corresponding TPE/SBS blends due to the different morphology of the supporting polymer matrix. Fluorescence domains are actually randomly distributed and less contrasted in TPE/SBR, while they appear to coalesce each other in TPE/SBS, giving rise to broader, island-like features due to the block morphology.

Further study is extended to other polymeric materials and fluorescent molecular rotors in order to validate the discovered method as a new tool for polymer traceability. 


\section{Acknowledgements}

This work has been supported by the Fondazione Cassa di Risparmio di Pisa under "POLOPTEL" project no. 167/09.

\section{References}

1. S. Banerjee, A. K. Tyagi and Editors, Functional Materials: Preparation, Processing and Applications, Elsevier Inc., Waltham, MA, USA, 2012.

2. X. D. Liu, A. R. Esker, M. Haeussler, C. Kim, P. Lucas, M. Matsunaga, N. Nishi, J. J. Robin, B. Z. Tang, D. A. Wang, M. Yamada and H. Yu, Functional Materials and Biomaterials. [In: Adv. Polym. Sci., 2007; 209], Springer-Verlag Berlin, Heidelberg, 2007.

3. J. M. G. Cowie and V. Arrighi, Polymers: Chemistry and Physics of Modern Materials, CRC Press, Boca Raton, FL, 2008.

4. F. Ciardelli, G. Ruggeri and A. Pucci, Chemical Society Reviews, 2013, 42, 857-870.

5. P. Minei, A. Battisti, S. Barondi, M. Lessi, F. Bellina, G. Ruggeri and A. Pucci, ACS Macro Letters, 2013, 2, 317-321.

6. B. Makowski, J. Kunzelman and C. Weder, in Handbook of Stimuli-Responsive Materials, ed. M. W. Urban, Wiley-VCH Verlag GmbH \& Co. KGaA, Weinheim, Germany., 2011, pp. 117-138.

7. A. Pucci and G. Ruggeri, Journal of Materials Chemistry, 2011, 21, 8282-8291.

8. A. Pucci, R. Bizzarri and G. Ruggeri, Soft Matter, 2011, 7, 3689-3700.

9. J. B. Birks, Photophysics of Aromatic Molecules Wiley-Interscience, London, 1970.

10. Y. Hong, J. W. Y. Lam and B. Z. Tang, Chem. Commun., 2009, 4332-4353.

11. Y. Hong, J. W. Y. Lam and B. Z. Tang, Chemical Society Reviews, 2011, 40, 5361-5388.

12. A. Pucci, R. Rausa and F. Ciardelli, Macromol. Chem. Phys. , 2008, 209, 900-906.

13. Z. Chi, X. Zhang, B. Xu, X. Zhou, C. Ma, Y. Zhang, S. Liu and J. Xu, Chemical Society Reviews, 2012, 41, 3878-3896.

14. A. Qin, J. W. Y. Lam and B. Z. Tang, Progress in Polymer Science, 2012, 37, 182-209.

15. X. Zhang, Z. Chi, Y. Zhang, S. Liu and J. Xu, Journal of Materials Chemistry C: Materials for Optical and Electronic Devices, 2013, 1, 3376-3390.

16. Z. Zhao, J. W. Y. Lam and B. Z. Tang, Journal of Materials Chemistry, 2012, 22, 2372623740.

17. Z. Zhao, J. W. Y. Lam and B. Z. Tang, Soft Matter, 2013, 9, 4564-4579.

18. N. B. Shustova, B. D. McCarthy and M. Dinca, Journal of the American Chemical Society, 2011, 133, 20126-20129.

19. J. Zhou, Z. Chang, Y. Jiang, B. He, M. Du, P. Lu, Y. Hong, H. S. Kwok, A. Qin, H. Qiu, Z. Zhao and B. Z. Tang, Chemical Communications, 2013, 49, 2491-2493.

20. G.-J. Zhao, K.-L. Han, Y.-B. Lei and Y.-S. Dou, Journal of Chemical Physics, 2007, 127, 094307/094301-094307/094306.

21. P. F. Barbara, S. D. Rand and P. M. Rentzepis, Journal of the American Chemical Society, 1981, 103, 2156-2162.

22. C. L. Schilling and E. F. Hilinski, Journal of the American Chemical Society, 1988, 110, 2296-2298.

23. Q. Chen, J.-X. Wang, F. Yang, D. Zhou, N. Bian, X.-J. Zhang, C.-G. Yan and B.-H. Han, Journal of Materials Chemistry, 2011, 21, 13554-13560.

24. R. Hu, J. W. Y. Lam, J. Liu, H. H. Y. Sung, I. D. Williams, Z. Yue, K. S. Wong, M. M. F. Yuen and B. Z. Tang, Polymer Chemistry, 2012, 3, 1481-1489. 
25. R. Hu, J. L. Maldonado, M. Rodriguez, C. Deng, C. K. W. Jim, J. W. Y. Lam, M. M. F. Yuen, G. Ramos-Ortiz and B. Z. Tang, Journal of Materials Chemistry, 2012, 22, 232-240.

26. W. Wu, S. Ye, L. Huang, L. Xiao, Y. Fu, Q. Huang, G. Yu, Y. Liu, J. Qin, Q. Li and Z. Li, Journal of Materials Chemistry, 2012, 22, 6374-6382.

27. W. Wu, S. Ye, R. Tang, L. Huang, Q. Li, G. Yu, Y. Liu, J. Qin and Z. Li, Polymer, 2012, 53, 3163-3171.

28. C. E. Bunker, N. B. Hamilton and Y. P. Sun, Analytical Chemistry, 1993, 65, 3460-3465.

29. M. Kaholek, P. Hrdlovic and J. Bartos, Polymer, 1999, 41, 991-1001.

30. D. R. Lide, CRC Handbook of Chemistry and Physics, 86th Edition, CRC Press LLC, Boca Raton, FL, USA, 2004.

31. J. W. Gooch, Encyclopedic Dictionary of Polymers, 2nd Edition, Springer, Berlin, 2010.

32. L. C. Burmeister, Convective heat transfer, 2nd Edition, John Wiley \& Sons, Inc., New York, 1993.

33. I. G. A. Camposeo, F. Tantussi, S. Pagliara, M. Moffa, F. Fuso, M. Allegrini, E. Zussman and D. Pisignano, Nano Letters, in press.

34. F. Tantussi, S. Menghetti, E. Caldi, F. Fuso, M. Allegrini and G. Galli, Applied Physics Letters, 2012, 100.

35. K. Karrai and R. D. Grober, Applied Physics Letters, 1995, 66, 1842-1844.

36. M. Faraj, E. Elia, M. Boccia, A. Filpi, A. Pucci and F. Ciardelli, Journal of Polymer Science, Part A: Polymer Chemistry, 2011, 49, 3437-3447.

37. A. Pucci, C. Barsocchi, R. Rausa, L. D'Elia and F. Ciardelli, Polymer, 2005, 46, $1497-$ 1505.

38. E. Passaglia and F. Donati, Polymer, 2007, 48, 35-42.

39. J. Heimel, H. Fuchs and U. C. Fischer, Single Molecules, 2002, 3, 301-309.

40. D. Jarzab, M. T. Lu, H. T. Nicolai, P. W. M. Blom and M. A. Loi, Soft Matter, 2011, 7, 1702-1707.

41. N. Yamamoto, T. Mizokuro, H. Mochizuki, S. Horiuchi, T. Hayakawa and T. Hiraga, Journal of Microscopy-Oxford, 2004, 213, 135-139.

42. A. Ambrosio, M. Alderighi, M. Labardi, L. Pardi, F. Fuso, M. Allegrini, S. Nannizzi, A. Pucci and G. Ruggeri, Nanotechnology, 2004, 15, S270-S275.

43. S. Menghetti, M. Alderighi, G. Galli, F. Tantussi, M. Morandini, F. Fuso and M. Allegrini, Journal of Materials Chemistry, 2012, 22, 14510-14517. 
Supplementary information of the paper "Aggregation Induced Emission of Tetraphenylethylene Dyes into Styrene based Polymers", by A. Pucci et al.

Figure S1. Sketch of the SNOM optical setup

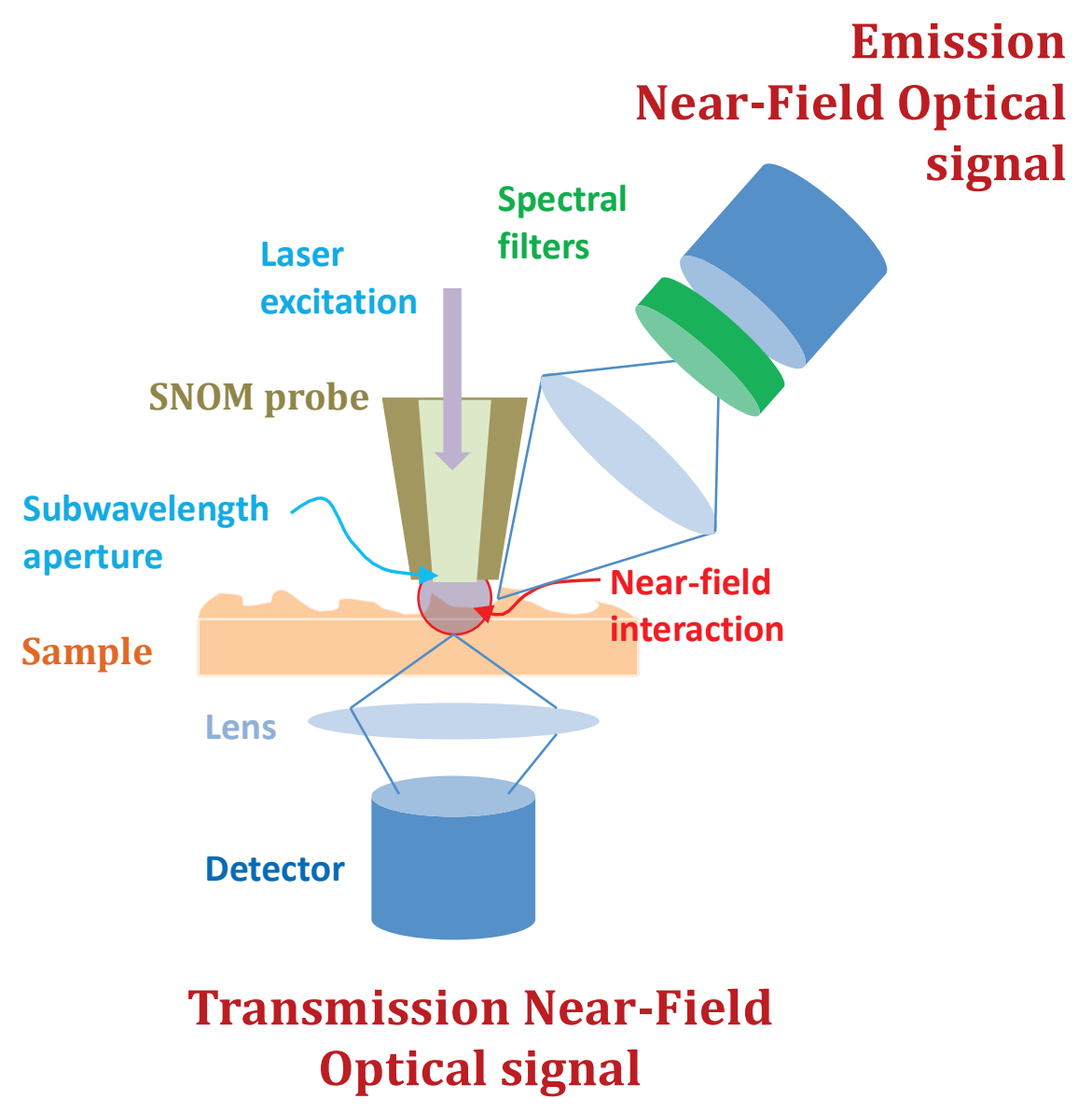


Figure S2. Energy diagram of the TPE photophysics

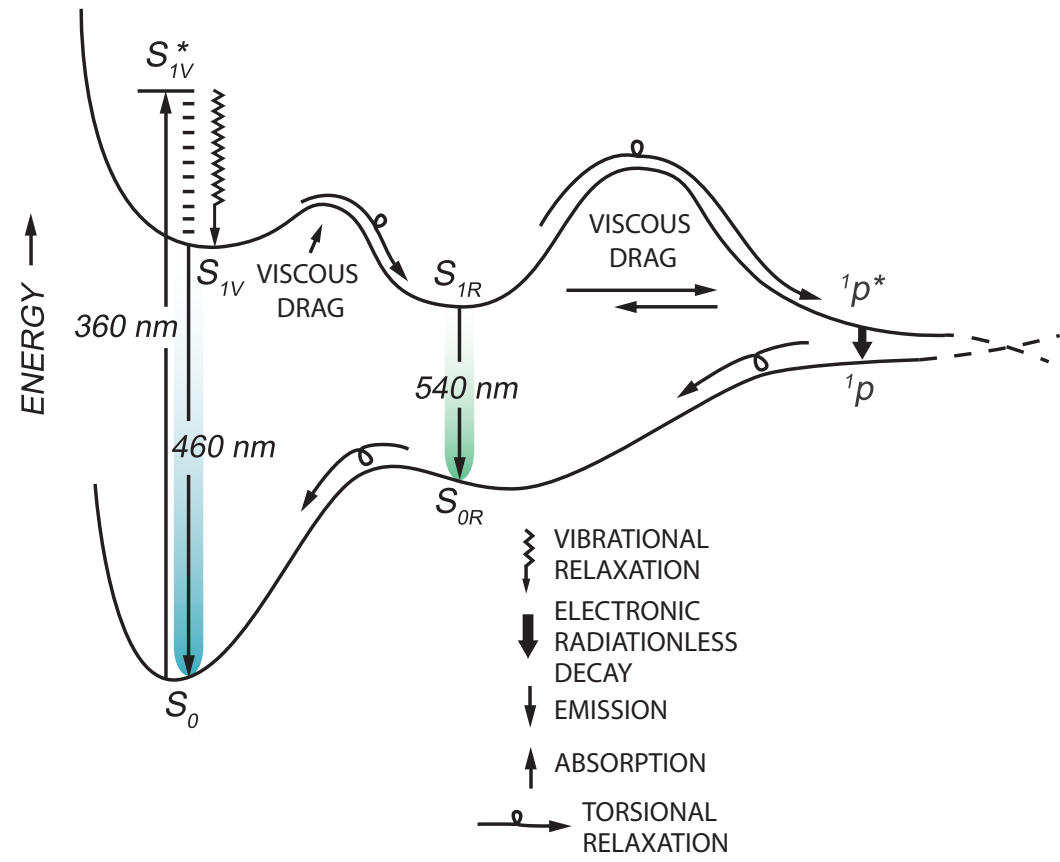

$\theta=0 \longleftarrow$ TWISTING ANGLE $\longrightarrow \quad \theta=\pi / 2$ 